\title{
S50. Structured diagnostic interviews
}

\section{STRUCTURED DLAGNOSTIC INTERVIEWS IN EPIDEMIOLOGY}

Hans-Ulrich Wittchen, Max Planck Institut für Psychiatrie, Klinisches Institut, Kraepelinstr.2, München, Germany

Unlike to the pre-1980 era, almost all epidemiological surveys since 1980 have been conducted with structured or even fully standardized diagnostic assessment instruments. Because most of these new instruments are diagnostically more comprehensive and specific, more time efficient (average of 1 hour administration time) and can be used even by non-clinicians, the design features of more recent studies have changed considerably. (1) the traditional 2-stage design, in which screening instruments are used first, followed by a clinicians reexamination is not used any more, except in a few stadies, (2) since less costly non-clinicians can be used, most epidemiological studies are now based on considerably larger samples, allowing the application of more sophisticated statistical analyses, (3) epidemiological studies on mental disorders are now able of covering a considerably larger spectrum of disorders, than previously.

There are at least four groups of diagnostic interviews. Most frequently fully standardized instruments such as the DIS or the CIDI are being used, less frequently more loosely structured interviews such as the PSE or derivations thereof. Few studies developed ad hoc instruments to cover specific diagnostic research interests, such as the SPIKE.

The paper discusses the characteristics and methodological findings of these instruments and emphasizes the importance of consistency and reliability of the assessment procedures.
STRUCTURED DIAGNOSTIC INTERVIEWS IN TRAINING IP Lépine.

Department of Psychiatry, Groupe Hospitalier Bichat - Claude Bernard, 46 rue H. Huchard, 75877, Paris, Cedex 18, France

The current classification of mental disorders and the reference to signs and symptoms used as diagnostic criteria has shed new light on the importance of the clinical interview process. In fact, the operationalization of these criteria and the definition of the algorithms generating diagnoses underline the fact that when defining questions to be used in a structured diagnostic interview, all ambiguities need to be solved. Some words used in the labelling of diagnostic criteria are far from being precise enough and their grammar, as stress by L. Robins, allows many different options to be made. In many cases, the translation criteria into questions process requires asking additive questions before one can say that a specified criterion is fullfilled.

During the training courses, several of these problems must be perceived by the trainees in order to improve their use of a diagnostic instrument. Moreover, general guidelines used in the training procedures and their application to trainees with different clinical experience can be put forward. The agenda of different training sessions of several instruments and their packages and guidelines are reported as well as the requirements for the participants to such courses. The use of these instruments in the field of general training of students and clinicians is emphazised in the perspective of an improvement of the diagnostic process in mental disorders. 
STRUCTURED DIAGNOSTIC INTERVIEWS IN CLINICAL RESEARCH José Guerrero

University of Sevilla, Avda Dr. Fedriani, E-41009 Sevilla, Spain

The construction of scales and questionnaires gives rise to complex methodological problems, such as the difficulty of ensuring a valid correspondence with nosological criteria and global diagnosis. In spite of extensive research in the last decade, structured instruments are not largely used in clinical research. Although reasons are not clear, it is likely that clinical research is based on non-structured global criteria, contrary to clinical trials or epidemiological research.

A review of the literature shows that structured instruments are most often used in research on drugs and alcohol. In addition, the need for structuralization is not the same for all current diagnostic criteria.

Our study shows that the need for structuralization is the. highest for personality disorders, particularly when young psychiatrists or psychiatrists in training are considered.
Structured Interviews for Primary Care
T.B. Ustün

World Health Organization, Division of Mental Health, Geneva, Switzerland

OBJECTIVES: This paper reviews the structured interviews for use in primary care for the diagnosis and classification of mental disorders.

METHODS: Several interviews used for research and clinical assessments in primary care settings are reviewed for their relevance to primary care, pscyhometric properties (reliability, validity, sensitivity and specificity against different measures), availability in different languages, ease of use in different primary care settings. RESULTS: The instruments used in primary care can be classified as (a) screening or case finding instruments (b) diagnostic interviews and (c) personal assessment instruments that assess problems, disablements environmental factors, (d) instruments that combine evaluation (e.g. diagnosis and treatment). Examples and basic features of instruments in each group are given . A: General Health Questionnaire[GHQ] and Self-Reporting Questionnaire [SRQ] Alcohol Use Disorders Identification Test [AUDIT]; $B$ : Composite Diagnostic Interview Schedule [CIDI] Primary Care Version, Primary Care Evaluation of Mental Disorders [Prime- MD]; Symptom Driven Diagnostic System [SDDS]; C: Social Disability Schedule [SDS], and Brief Disability Questionnaire [BDQ] ; $D$ : Primary Care Version of ICD-10 PHC which focusses on 24 most frequent conditions and matches the diagnostic guidelines with best possible management guidelines known. These instruments were used in different international studies with ease and satisfactory reliability. They were well accepted by patients indicating that they can be used for both research and clinical purposes.

CONCLUSION: Standardized assessment and management methods for people with psychological disorders in general health care are shown to be applicable in different cultures. Future research is needed how effective these methods are on the outcome of these disorders.
STRUCTURED DIAGNOSTIC INTERVIEWS IN PERSONALITY DISORDERS MC Pull. CB Pull

Service de Psychiatrie, Centre Hospitalier de Luxembourg, 4 rue Barblé, L-1210 Luxembourg, Luxembourg

Recent classification systems, such as the WHO'S ICD-10 and the APA's DSM, propose explicit diagnostic criteria for making a diagnosis of personality disorder. The classifications do not however provide guidelines for assessing those criteria in a valid and reliable way.

In recent years, several authors or organizations have developed structured clinical interviews for the assessment of personality disorders. In the present report, the authors intend to give a brief account on the best known of these instruments, and they will describe, in more detail, an instrument which has been developed by the Worid Health Organization : the International Personality Disorder Examination or IPDE.

The IPDE is a semi-structured diagnostic interview for the assesment of personality disorders according to ICD-10, DSM-III and DSM-IV criteria. The instrument has been field tested in the International Pilot Study of Personality Disorders. The IPDE was administered to 716 patients enrolled in clinical facilities at 14 participating centers located in 11 countries in North America, Europe, Africa and Asia. The interview proved to be acceptable to clinicians, and it demonstrated an interrater reliability and temporal stability comparable to that reported for well established instruments used to diagnose schizophrenia and the mood and anxiety disorders. 